contact) but rather to the supply of medium (Nature, 246, 197; 1973). These experiments implied that cells stop growing when the medium is exhausted. But this does not explain two important observations. The first is that medium removed from contact-inhibited cultures will support the growth of nonconfluent cells, showing that the medium is not exhausted. Second, if a strip of cells is removed from a confluent contact-inhibited culture, the cells at the edge of the 'wound' and those that migrate into the denuded area will divide, while the cells in the confluent cell layer which share the same culture medium will not. The 'wound' experiment was seen as the most persuasive evidence that cell-cell contact itself plays a part in contact inhibition and that contact somehow desensitises the cells to growthpromoting factors in the medium.

How does this contact-induced desensitisation occur? A possibility originally proposed by Rubin and expounded by Stoker (Nature, 246, 200; 1973) is that there is a layer of medium in close contact with the cells which does not exchange freely with the bulk of the culture medium. Access of the cell layer to medium components would be limited by diffusion across this boundary layer and this limitation would lead to contact inhibition.

The diffusional flux of medium solutes across this boundary layer may be increased in two ways. One is to increase their concentration in the bulk of the medium (which would explain why the addition of increased concentrations of serum will stimulate growth in contact-inhibited cells), the second is to decrease the depth of the diffusion boundary layer. This vanishes at the edge of the cell sheet (which explains why cells in the wound can divide while those in the cell layer do not). Also the depth of the layer can be decreased by increasing the velocity of medium flow over the cell sheet: a fourfold increase in velocity will double the diffusional flux across the boundary layer-effectively doubling the concentration of medium factors reaching the cell surface (Maroudas, Cell, 3, 217 ; 1974). This interpretation of the mechanism of contact inhibition was supported when Stoker showed that pumping medium rapidly across a confluent cell sheet will promote some cell division along the medium stream. In a more recent paper Stoker shows that the vast majority of contact-inhibited cells can be induced to divide without changing the medium (Cell, 3, 207; 1974) if the velocity of medium flow across a cell layer is greatly increased by vigorous shaking of the culture.

The question that remains is: what are the crucial medium factors needed by the cell to promote cell division?
This depends on the cell type and the conditions of culture but in cases where the bulk of the medium is not depleted, diminished access to serum factors across the boundary layer is probably the cause of contact inhibition. The fact that many tumour cells do not exhibit contact inhibition may be ascribed to their relative insensitivity to depletion of serum in the culture medium, so that even at high cell densities diffusion across the boundary layer is sufficient to provide the small amounts of serum necessary for tumour cell growth. If this was the case then tumour cells selected for inability to grow in low concentrations of serum should become contact inhibited. This has been shown to be the case (Vogel and Pollack, J. cell. comp. Physiol., 82, 189; 1973).

So it seems that contact inhibition (at least in mouse fibroblasts) may be explained by the existence of a diffusion boundary layer that prevents ready access to serum factors in the culture medium rather than by cell-cell contact. Whether serum sensitivity will replace contact inhibition as the new criterion for the normality of cells remains to be seen.

\section{Terns as predators}

from our Animal Ecology Correspondent THE classic model of predator-prey interaction proposed by Volterra (Mem. Acad. naz. Lincei (ser 6), 2, 31; 1926) has been criticised on many grounds. Most criticisms have centred on the biologically impossible constraints concerning the reproductive rates of both predator and prey and their movements in time and space. In real life the dynamics of interactions are influenced by many factors such as a sharply defined breeding season and a time lag between the consumption of prey and the production of young as a response to it by the predator.

Maynard Smith and Slatkin have drawn attention to the likelihood that stability in predator-prey interactions is strongly influenced by yet another factor, namely the variety of hunting abilities normally found in mixed-age populations of predators (Ecology, 54, $384 ; 1973$ ). According to their calculations stability is maintained by the conservation of a prey species brought about by the strongly selective effects of the density of both it and its predator, and the differential in hunting ability found within the predator population. There is little doubt that the young of some species are totally ineffective hunters until they have been taught all the finer points of stalking. Remarkably little is known about the ecological effects on the prey species of such a life history strategy even with respect to the best documented species.
It is interesting, therefore, to note the work of Buckley and Buckley on the feeding ecology of the royal tern, Sterna maxima (Ecology, 55, 1053; 1974).

Working in the Netherlands Antilles islands where royal terns spend the winter, Buckley and Buckley recorded data on several aspects of feeding behaviour. First, they observed as many dives as practicable and noted whether or not they resulted in fish being caught. If fish were dropped after being caught, this too was recorded. Second, they recorded the time budget of birds by counting the numbers of adults and juveniles on the roosts. Subtraction of this from the total number gave a measure of the time spent fishing. Third, they recorded the length of the periods when adults were fishing alone, juveniles fished alone, or both age classes together. Their results showed that adults' foraging passes lasted about half as long as did those of juveniles. Adults made 1.7 times more dives per minute as juveniles and the number of fish eaten per minute was 1.6 times that of juveniles. This was not because juveniles obtained fewer fish per dive than adults-although they frequently dropped, but recaught, the fish-but that their diving rate was lower. These observations are consistent with the notion that fish are difficult to catch but that an increased fishing rate can offset this difficulty. The relative absence of adult terns, but not juveniles, from areas low in fish suggests further that adults are more adept at detecting prey than juveniles, and so work to a more relaxed time budget.

Some years ago Salt and Willard attempted a component analysis of predation of Forster's tern but did not, unfortunately, compare adult with juvenile fishing success (Ecology, 52, $989 ; 1971)$. They suggested that searching rate by birds is inversely proportional to prey density. In the light of the Buckleys' study this should be modified to include 'within age classes'. The drop in attack rate in early spring noted by Salt and Willard was probably the result of the sudden influx of juveniles into the population and the rise of successful captures from spring through to winter an expression of a change in the structure of the prey population

The royal tern study has demonstrated clearly that several foraging variables are subject to change with respect to age of individual and associated age structure of the predator population. Little by little reliable data are emerging which show that the accepted axioms of the Volterra approach are in need of revision. As far as terns are concerned, the cheeping of tiny beaks may be a blessing in disguise to later population stability. 